\title{
T-shaped mode coupler for two-mode mode division multiplexing
}

\author{
Hirokazu Kubota ${ }^{\text {a) }}$, Hidehiko Takara, and Toshio Morioka \\ NTT Network Innovation Laboratory, NTT Co., 1-1 Hikarino-oka, Yokosuka, \\ Kanagawa, 2390847, Japan \\ a)kubota.hirokazu@lab.ntt.co.jp
}

\begin{abstract}
We propose a novel type of mode coupler consisting of two identical rectangular few-mode waveguides with different orientations. Numerical simulation indicates that mode extinction ratio exceeding $20 \mathrm{~dB}$ can be achieved by well-aligned waveguides.
\end{abstract}

Keywords: few mode fiber, PLC, mode coupler, mode division multiplexing.

Classification: Optoelectronics, Lasers and quantum electronics, Ultrafast optics, Silicon photonics, Planar lightwave circuits

\section{References}

[1] D. Oian, M.-F. Huang, E. Ip, Y.-K. Huang, Y. Shao, J. Hu, and T. Wang, "101.7-Tb/s $(370 \times 294 \mathrm{~Gb} / \mathrm{s})$ PDM- 128QAM-OFDM transmission over $3 \times 55-\mathrm{km}$ SSFM using pilot-based phase noise mitigation," Optical Fiber Communication Conference 2011, Los Angels, USA, PDP B5, March 2011.

[2] J. Sakaguchi, Y. Awaji, N. Wada, A. Kanno, T. Kawanichi, T. Hayashi, T. Taru, T. Kobayashi, and M. Watanabe, "109 Tb/s $(7 \times 97 \times 172-G b / s$ SDM/WDM/PDM) QPSK Transmission through 16.8-km homogeneous multi-core fiber," Optical Fiber Communication Conference 2011, Los Angels, USA, PDP B6, March 2011.

[3] N. Hanzawa, K. Saitoh, T. Sakamoto, T. Matsui, S. Tomita, and M. Koshiba, "Demonstration of mode-division multiplexing transmission over $10 \mathrm{~km}$ two-mode fiber with mode coupler," Optical Fiber Communication Conference 2011, Los Angels, USA, OWA4, March 2011.

[4] K. Imamura, K. Mukasa, and T. Yagi, "Investigation on multi-core fibers with large $A_{\text {eff }}$ and low micro bending loss," Optical Fiber Communications Conference 2010, San Diego, USA, OWK6, March 2010.

[5] T. Hayashi, T. Nagashima, O. Shimakawa, T. Sasaki, and E. Sasaoka, "Crosstalk variation of multi-core fibre due to fibre bend," ECOC 2010, Turin, Italy, We.8.F.6, Sept. 2010.

[6] A. R. Shah, R. C. J. Hsu, A. Tarighat, A. H. Sayed, and B. Jalali, "Coherent Optical MIMO (COMIMO)," J. Lightwave Technol., vol. 23, no. 8, pp. 2410-2419, Aug. 2005.

[7] M. Greenberg and M. Nazarathy, "Data parallelization by optical MIMO transmission over multimode fiber with intermodal coupling," J. Lightwave Technol., vol. 25, no. 6, pp. 1503-1514, June 2007.

[8] C. Koebele, M. Salsi, D. Sperti, P. Tran, P. Brindel, H. Mardoyan, S. Bigo, A. Boutin, F. Verluise, P. Sillard, M. Astruc, L. Provost, F. Cerou, and G. Charlet, "Two mode transmission at $2 \times 100 \mathrm{~Gb} / \mathrm{s}$, over $40 \mathrm{~km}-l o n g$ proto- 
type few-mode fiber, using LCOS-based programmable mode multiplexer and demultiplexer," Optics Express, vol. 19, no. 17, pp. 16593-16600, Aug. 2011.

[9] S. Randel, R. Ryf, A. Sierra, P. J. Winzer, A. H. Gnauck, C. A. Bolle, R.-J. Essiambre, D. W. Peckham, A. McCurdy, and R. Lingle, "6×56$\mathrm{Gb} / \mathrm{s}$ mode-division multiplexed transmission over 33-km few-mode fiber enabled by $6 \times 6$ MIMO equalizatio," Optics Express, vol. 19, no. 17, pp. 16697-16707, Aug. 2011.

\section{Introduction}

Optical communication systems have long been based on single-mode fibers (SMFs) to eliminate the bandwidth limitation imposed by the intermodal dispersion of multi-mode fibers (MMFs). With dense wavelength division multiplexing (DWDM) technology and advanced modulation formats, the transmission capacity of SMF has now exceeded $100 \mathrm{Tbit} / \mathrm{s}$ [1]. The next approach for expanding transmission capacity is based on space division multiplexing $[2,3]$. Two kinds of concepts have been studied as regards the space division multiplexing of optical fiber communication systems. One uses multi core fiber $[4,5]$ where a fiber consists of a number of single mode cores. The other is mode division multiplexing (MDM) $[6,7,8,9]$ where each fiber mode acts as a different data channel. Both techniques have coupled and uncoupled regimes. In coupled transmissions, transmitted multiple data signals can be mixed during propagation and a mathematical procedure such as MIMO must be incorporated to separate the signals. In uncoupled transmissions, the multiplicity is limited to ensure that no mode coupling occurs. The coupling may occur not only at transmission fibers but also at couplers and connectors. In spite of these limitations, the uncoupled system has an advantage in that a conventional transmission scheme, without signal processing, can be applied in each channel [4]. Polarization maintaining flat-core few-mode fiber (FMF) is a potential uncoupled transmission line. In this paper we propose a T-shaped mode coupler for two mode MDM, consisting of two identical flat few-mode waveguides. It acts as a directional coupler for the fundamental mode as usual. But it does not affect the transmission of $E_{12}$ and $E_{21}$ modes because mode overlapping between waveguides is negligible in these modes.

\section{Waveguide Structure}

Figure 1 (a) shows a schematic cross section of the proposed coupler. In this coupler, two identical rectangular few-mode waveguides are placed in a T-shape. Figure 1(b) shows the top view of the coupler. It has two input ports, ports 1 and 2, and two output ports, ports 3 and 4 . The mode multiplexed input signal from two mode fiber, which consists of $L P_{01}$ and $L P_{11}$ modes, is fed into one of the two input ports. The $L P_{01}$ mode couples to $E_{11}$ mode (fundamental mode) of the waveguide. Two components of $L P_{11}$ mode, $L P_{11 x}$ and $L P_{11 y}$ modes, couples to $E_{12}$ and $E_{21}$ modes, respectively. 
Dimension of the waveguides are $12 \times 16 \mu \mathrm{m}$ and a relative index $\Delta$ of $0.3 \%$ to supports $E_{11}, E_{12}$ and $E_{21}$ modes at an operating wavelength $\lambda$ of $1.55 \mu \mathrm{m}$. After propagating an appropriate distance, the fundamental mode component of the input signal is transferred to the opposite waveguide as shown in Fig. 1 (b), and it appears at port 3 as an output signal. The rest of the input signal components remain in the first waveguide, and appear at port 4 as an output signal.

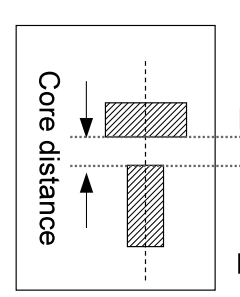

(a)

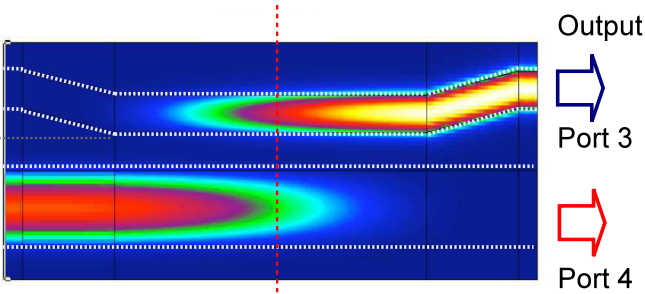

(b)

Fig. 1. Schematic drawing of T-shaped mode coupler. (a) Top view and (b) cross section.

\section{Numerical results}

First, we investigate the coupling strength change induced by the core orientation. Simulations were performed using FIMMPROP ver. 4. Here we neglected the propagation loss and nonlinearity, and set the operating wavelength at $1.55 \mu \mathrm{m}$. We adopted the effective index difference as a measure of the coupling strength. Each core has the same optical property; the effective index is the same even if there is no mode coupling. When two waveguides are placed near each other, the same mode of each waveguide forms a coupled mode. The coupled mode supports even and odd modes, each of which has a slightly different effective index, and strong coupling results in large index difference.

Figure 2 shows dependence of the coupling strengths of the waveguide modes on the core distance for three different core orientations. The solid, dotted and broken lines indicate T-shape, parallel, and in-line orientations, respectively. The symbol shapes indicate the mode number where the circles, squares, and triangles correspond to the $E_{11}, E_{12}$ and $E_{21}$ modes, respectively. With parallel and inline orientations, the coupling strengths of the $E_{12}$ and $E_{21}$ modes are stronger than that of the $E_{11}$ mode because these modes have broader spatial distributions than the $E_{11}$ mode. The coupling strength of the $E_{11}$ modes is almost independent of the core orientation, where as those of the $E_{12}$ and $E_{21}$ modes are more than 5 times less for the T-shape orientation than for the parallel orientation. As the modal profiles of the $E_{12}$ and $E_{21}$ modes have odd symmetries as shown in Fig. 2 (b) and (c), the field strength along one of the perpendicular bisectors of the waveguide is zero. Thus the coupling strength is reduced by the T-shaped orientation. The coupling length of each mode is estimated by $L_{c}=\lambda / 2\left(n_{e}-n_{o}\right)$. The $L_{c}$

(c) IEICE 2011

DOI: $10.1587 /$ elex.8.1927 Received September 25, 2011 Accepted October 20, 2011 Published November 25, 2011 


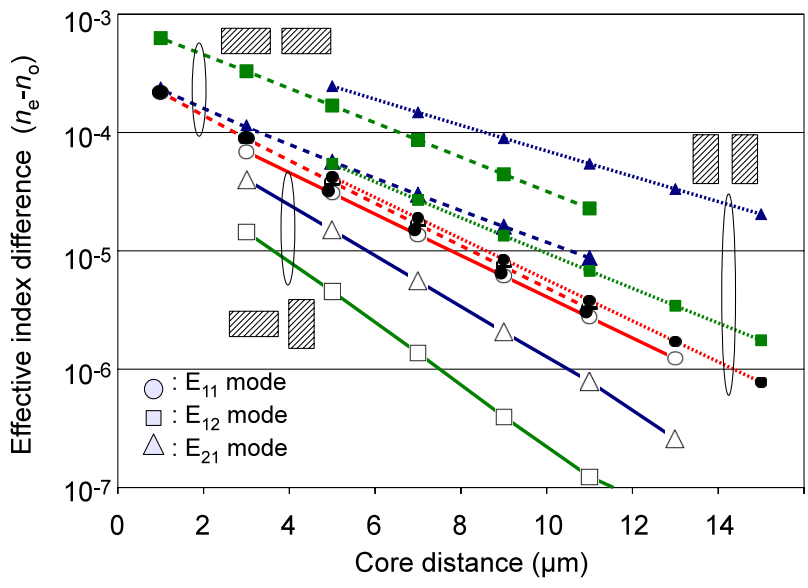

(a)

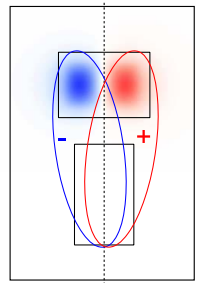

(b)

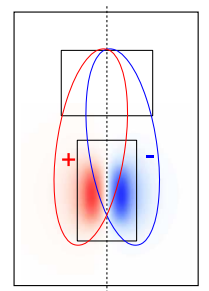

(c)

Fig. 2. (a) Effective index difference of three propagation modes along with core distance for three core orientations. Circles: $E_{11}$ mode, squares: $E_{12}$ mode, triangles: $E_{21}$ mode. (b), (c) Schematic mode profiles of the $E_{12}$ and $E_{21}$ modes.

values of the $E_{11}, E_{12}$ and $E_{21}$ modes calculated from Fig. 2 are 25.3, 170.6, and $51.8 \mathrm{~mm}$, respectively.

Figure 3 shows the transmission characteristics of the proposed coupler as a function of device length and core misalignment. The core distance is set at $7 \mu \mathrm{m}$ and the device length includes $5 \mathrm{~mm}$-long tapered waveguide sections at the input and output. The thick blue line in Fig. 3 (a) indicates the coupling characteristics of the fundamental mode with port 3. Almost $100 \%$ coupling was obtained by adjusting the device length. The thin blue and dotted blue lines indicate the coupling characteristics of the $E_{12}$ and $E_{21}$ modes to port 3 . These components cause the modal crosstalk of port 3 . The figure indicates that the crosstalk is less than $-30 \mathrm{~dB}$ at the optimum device length of $32.2 \mathrm{~mm}$. The red dotted and red dashed lines indicate the coupling characteristics of the $E_{11}$, and $E_{12}$ and $E_{21}$ modes with port 4, respectively. The intensity is almost constant for the $E_{12}$ and $E_{21}$ modes. The intensity of the $E_{11}$ mode is more than $30 \mathrm{~dB}$ less than that of the $E_{12}$ and $E_{21}$ modes at the optimum device length. This means the mode crosstalk in port 4 is also better than $-30 \mathrm{~dB}$. The coupling length of the fundamental mode obtained from Fig. 3 (a) is $25.1 \mathrm{~mm}$, which is consistent with that obtained from Fig. 2. It should be noted that no mode coupling occurs in the $E_{12}$ and $E_{21}$ modes despite the fact that the coupling length estimated from Fig. 2 is finite. Because of the odd symmetric mode profile, the coupling from each half is canceled out in T- shaped orientations, which means that the overlap integral is very small.

As the mode selection mechanism of the coupler is based on the antisymmetric mode profile of high order modes, the extinction ratio depends on the alignment of the two waveguides as shown in Fig. 3 (b). The coupling length of the $E_{11}$ mode shown by the black line is affected little by a small 


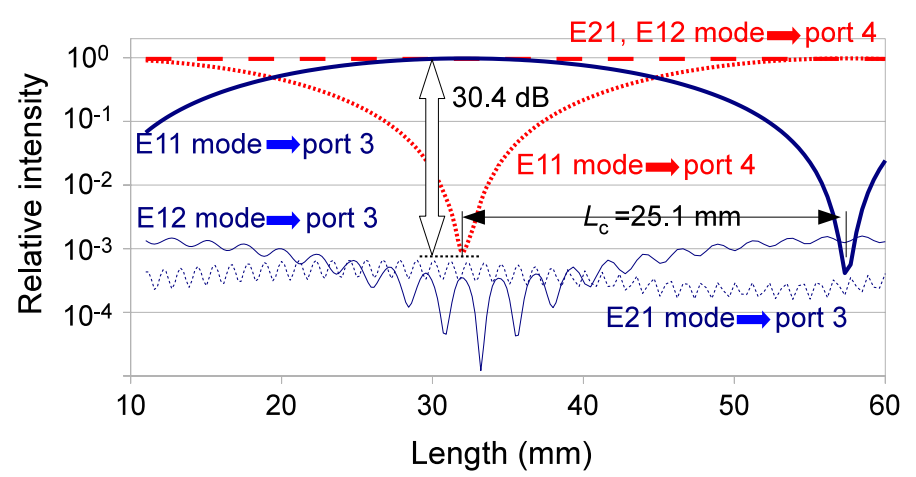

(a)

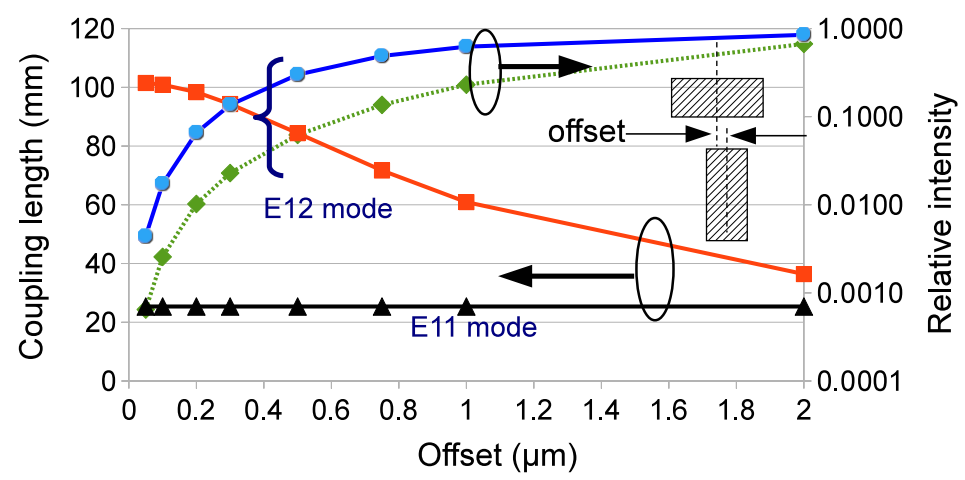

(b)

Fig. 3. Transmission characteristics of T-shaped mode coupler. (a) Transmittance as a function of device length for ideal core alignment. (b) Relative intensity (blue line) and coupling length (red line) of the E12 mode as a function of the core offset. The green broken line indicates the estimated crosstalk at a device length of $32.2 \mathrm{~mm}$. The coupling length of the E11 mode (black line) is also shown in the figure. Input signals from two-mode fiber were fed into port 2.

misalignment. The coupling length of the $E_{12}$ mode (red line) decreases when we increase the offset amount. As the power exchange between two waveguides is the sinusoidal dependence of the device length $z$ of $\sin ^{2}\left(\pi z / 2 L_{c}\right)$, we evaluated the crosstalk at the optimum device length (broken green line) from the peak coupled power (blue line) and the coupling length. The green line indicates that the two waveguides must align with an accuracy of better than $0.2 \mu \mathrm{m}$, or $1 \%$ of the long side of the waveguide cross-section, to obtain a $20 \mathrm{~dB}$ extinction ratio.

\section{Conclusion}

We proposed a novel mode coupler based on the spatial mode profile difference between the fundamental and high order modes. This coupler works as a directional coupler only for the fundamental mode when the waveguide supports up to three modes. A numerical simulation indicated that well-aligned waveguides can achieve a mode extinction ratio of more than $30 \mathrm{~dB}$. 


\section{Acknowledgments}

Part of this research was executed under the Commissioned Research of National Institute of Information and Communications Technology (NICT). 